\title{
INFLUENCE OF ECCENTRICITY ON ROUGHNESS DISTRIBUTIONS IN SIDE MILLING \\ (INFLUENCE OF ECCENTRICITY ON ROUGHNESS IN SIDE MILLING)
}

Irene Buj-Corral*, Joan Vivancos-Calvet, Hernán González-Rojas

Department of Mechanical Engineering. Universitat Politècnica de Catalunya.

Barcelona. Spain

Corresponding author. Avda. Diagonal, 647. 08028. Barcelona. Spain.

Tel: +34 934054016, Fax: +34 934016693, e-mail address: irene.buj@upc.edu

(Irene Buj-Corral)

Other authors: Joan Vivancos-Calvet (joan.vivancos@upc.edu), Hernán González-

Rojas (hernan.gonzalez@upc.edu) 


\begin{abstract}
Herein is reported a study on the influence of eccentricity on the confidence intervals of roughness distributions obtained in side milling processes, for families of tools having a certain value of runout or tool grinding error. A model was previously developed to predict roughness along a line in the feed direction. It is based on the geometric intersection of the piece and the tool, and enables determination of a roughness profile as a function of feed, tool radii and eccentricity E. Arithmetic average roughness $R_{a}$ and peak-to-valley roughness $R_{t}$ were obtained for the tools within a family. Each family is defined by an average radius value and a standard deviation value of all tool radii. The Monte Carlo method was employed to generate $\mathrm{N}$ random combinations of radius values for each family, according to a normal distribution. The model was validated with experimental tests. For each family of tools roughness distributions were obtained at different feed values and for different eccentricity values. It was found that the higher eccentricity, the closer to the upper reference value the median and mode of the roughness distribution are, with more asymmetrical roughness distributions. Effect of eccentricity on roughness distribution is more remarkable at low than at high runout values.
\end{abstract}

Keywords: side milling; grinding errors; eccentricity; Monte Carlo; surface roughness 


\section{INTRODUCTION}

Side milling is usually employed to manufacture molds and dies — namely, to obtain vertical walls in contours, cavities or pockets. Further finishing operations are grinding, electrical discharge machining (EDM), or manual polishing (Altan et al., 2001). In order to minimize polishing time and costs, low roughness is required in the previous milling operation.

Roughness of a milled surface depends partly on cutting conditions, especially feed, and partly on geometric factors of the tool, such as its radius; grinding errors (which lead to differences in the cuttings edges radii); eccentricity (also known as tool parallel axis offset); axis inclination (tilt angle); tool deflection; or wear (Spiewak, 1995). It also depends on external loads, fixture stiffness, contact interaction between tool and workpiece, as well as tool material and workpiece material (Arrazola et al., 2013).

Eccentricity occurs when the tool's rotational axis does not coincide with its geometric axis. Various authors have studied milling processes in which the tool shows eccentricity or tool axis offset. (Kline et al., 1982) studied the effect of eccentricity and tilt on the surface profiles obtained in side milling. In that work they reported the appearance of heterogeneity bands due to parallel axis offset. They later studied the effect of eccentricity on chip thickness and cutting forces in end milling, by taking into account the effective radius of each tooth (Kline and DeVor, 1983). (Fu et al., 1984) extended the model and considered tool eccentricity, tool tilt and runout. (Ismail et al., 1993) predicted roughness in peripheral milling by considering flank wear, radial runout or eccentricity and tool vibrations. They also found heterogeneity bands on the workpieces' surface along the axial direction. (Ranganath and Sutherland, 2002) 
developed a model for predicting tool radii and cutting forces in end milling, which included tool grinding errors, parallel axis offset and tilt. (Wang and Zheng, 2003) reported an analytical method to identify the 'offset geometry' in end milling from the measurements of the end milling force. For end milling (Ryu et al., 2006) obtained surface topographies in end milling considering errors produced not only by tool runout, tool eccentricity and tilt, but also tool deflection caused by cutting forces and back cutting of teeth. They observed that tool runout and tilt strongly influenced roughness parameters such as RMS or root mean squared, skewness and kurtosis, while eccentricity had little effect on them. (Schmitz et al., 2007) studied the effect of eccentricity on roughness profiles obtained in end milling. They observed that high eccentricity values led to profiles with constant special period, since only one of the cutting teeth of the milling tool leaves a mark on the workpiece's surface. (Omar et al., 2007) studied the effects of the tool tilt, eccentricity, deflection and wear, and the effects of the machine-tool-workpiece dynamics, on the surface roughness obtained from side milling with a cylindrical mill. These authors all observed that eccentricity leads to higher surface roughness, which results from one of the cutting edges removing more material than the other ones, in a scenario resembling milling with only one tooth per revolution. Considering tool setting error, which contains both parallel axis offset and tilt, (Arizmendi et al., 2009) observed that width of heterogeneity increases with tool setting error, while (Buj-Corral et al., 2011) found that width decreases with helix angle of the milling tool.

Although several authors have studied surface profiles and topographies obtained in side milling processes when considering tool runout, only few of them have taken into account roughness distributions to be obtained when a family of tools having different tool radii are studied. For example, (Franco et al., 2004) simulated roughness to be 
obtained for different tools considering axial and radial runout in face milling. (BujCorral et al., 2013) explored the roughness distributions for arithmetic average roughness Ra to be obtained for a family of tools having different combinations of tool grinding errors in peripheral milling. It was observed that the greater the variation among the radii, the larger the interval of possible roughness values. For a sufficiently high grinding error, the size of the interval increased with increasing feed, as did the mode or most frequent value of obtained roughness. The present work is an extension of the work by (Buj-Corral et al., 2013) in the study of roughness distributions when both runout due to tool grinding error and eccentricity due to parallel axis offset are considered.

\section{MODEL}

A computational program incorporating a series of algorithms to simulate a roughness profile in the feed direction was presented elsewhere (Buj-Corral et al., 2013), for a cylindrical milling tool in peripheral milling.

The effects of the tool geometry on the workpiece topography were determined at the intersection of each cutting edge with the material, moving along the piece at a defined feed rate and rotation speed. The tool edge trajectories were assumed to be circumferential rather than trochoidal, since cutting speed greatly exceeds feed rate.

In the program, $\mathrm{X}$ axis is discretized in increments $\Delta \mathrm{x}$. First, the path of the first tool tooth is determined by means of a circumferential trajectory around the geometric tool center. Then the geometric center of the tool moves a distance equal to feed per tooth $f$ $\left(\mathrm{mm} \cdot\right.$ tooth $^{-1} \cdot$ revolution $\left.^{-1}\right)$ and the path for the next tooth is determined by means of a 
circumferential trajectory around the geometric tool center, according to the toothmaterial intersection. This provides the successive arcs of the trajectories of the tooth ends, which intersect with each other leading to a roughness profile (Figure 1).

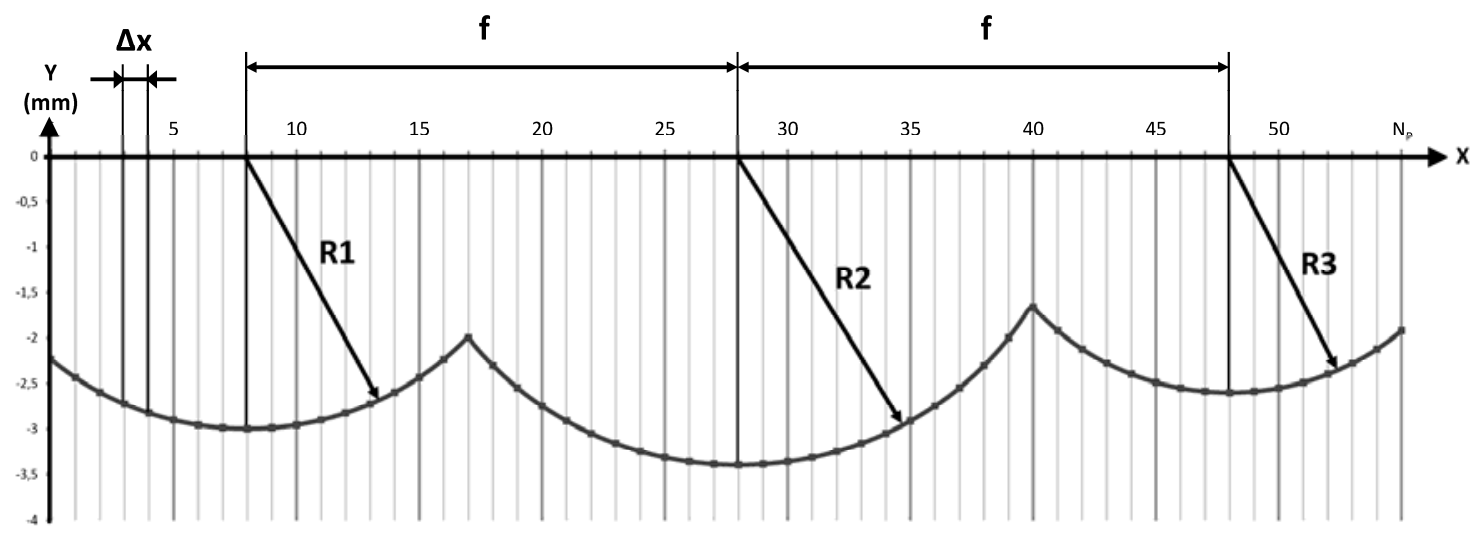

Figure 1

When no eccentricity is considered, the circular trajectory of the end of each tooth $k$ is given by Equation 1 (Kline et al., 1982).

$$
y_{i}=-\sqrt{R_{k}^{2}-x_{i}^{2}}
$$

Where $R_{k}$ is radius of each tool tooth $k$

$x_{i}$ is the $x$ position of each discrete point along axis $\mathrm{X}$, and

$y_{i}$ is the corresponding $y$ coordinate for each $x_{i}$ position

Each radius $R_{k}$ of the milling tool is considered to be different to the rest of the radii because of grinding errors.

Eccentricity or tool axis offset is defined as the distance between tool rotating axis and tool geometric axis. In order to add eccentricity to the previous model, then the rotation centre $\mathrm{O}$ of the milling tool teeth does not coincide with the geometric centre $\mathrm{C}$ of the milling tool (Figure 2). In this case, instantaneous effective rotation radius Rek of each 
tooth's $k$ end $\mathrm{P}$, is different than geometric radius $R k$. In addition, due to eccentricity, $R_{e k}$ is variable. Thus, for each tool tooth $k, R_{e k}$ will take a different value for each one of the $x_{i}$ positions of one tool end considered. In summary, when no eccentricity is considered, each tool has a different radius because of runout, but radius is kept constant. On the contrary, when eccentricity is considered, effective radius varies along $\mathrm{X}$ axis due to eccentricity.

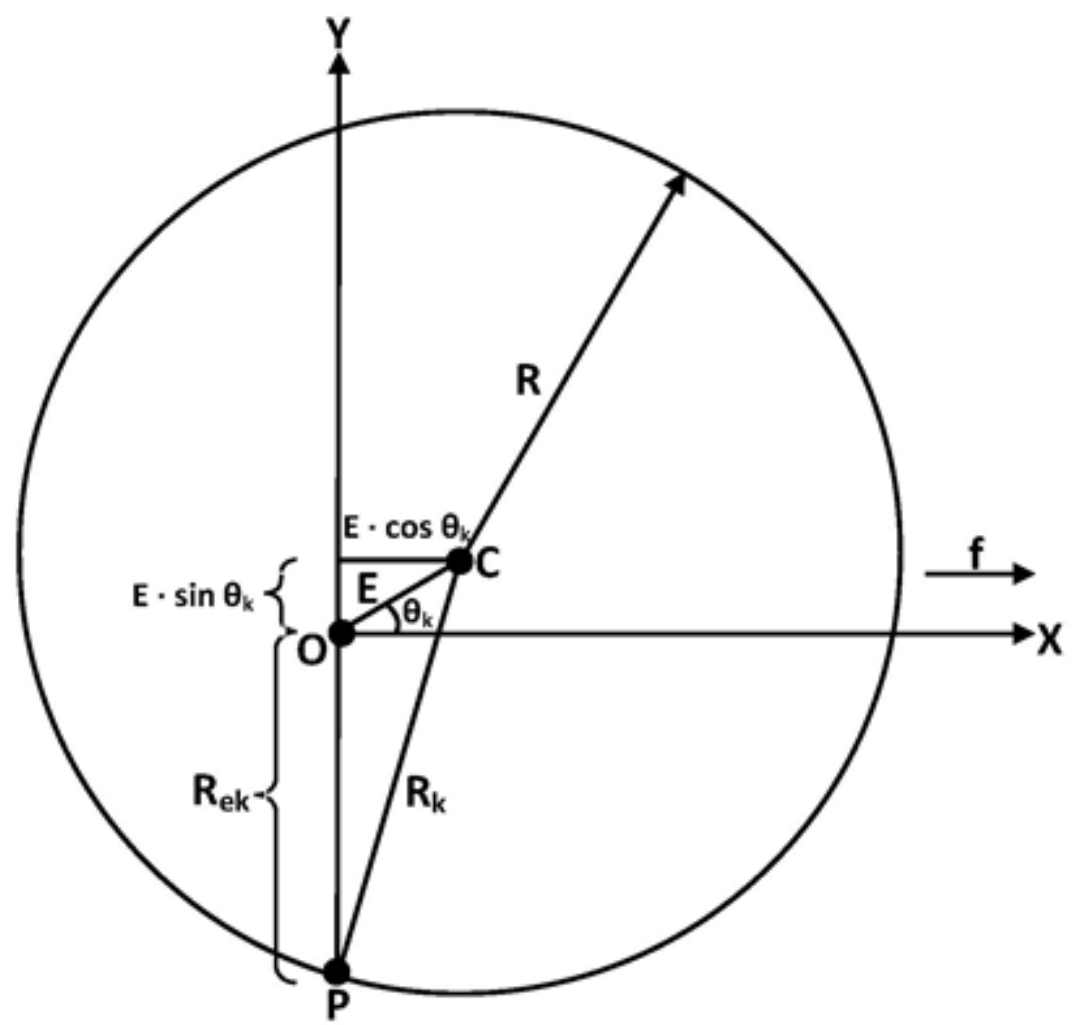

Figure 2

From Figure 2 Equation 2 is deduced. It gives the instantaneous value of effective cutting radius $R_{e k}$ of each tooth $k$ as a function of geometric radius $R_{k}$ of tooth $k$, of eccentricity $E$ and of position angle $\theta_{k}$, in a reference system XY that advances with the rotation center $\mathrm{O}$ of the milling tool and does not rotate. $\mathrm{X}$ axis is parallel to feed $f$.

$$
R_{e k}=-E \cdot \sin \theta_{k}+\sqrt{R_{k}^{2}-\left(E \cdot \cos \theta_{k}\right)^{2}}
$$


Angle $\theta_{k}$ positions eccentricity $E$ with respect to $\mathrm{X}$ axis when the end $\mathrm{P}$ of a tooth $\mathrm{k}$ coincides with negative $\mathrm{Y}$ axis. Between two consecutive teeth $k$ and $k+1$, increment of position angle $\theta_{k}$ is given by Equation 3 .

$$
\Delta \theta k=\frac{2 \pi}{n_{t}}
$$

Where $n_{t}$ is the number of tool teeth.

According to the methodology employed, coordinate $x$ is discretized in points that are separated a distance $\Delta x$, and position angle $\theta_{k}$ is discretized in increments $\Delta \theta_{k \mathrm{i}}$ as a function of $\Delta x$, according to equation (4).

$$
\Delta \theta_{k i}=\Delta x_{i} \cdot \frac{2 \pi}{n_{t}} \cdot \frac{1}{f}
$$

If eccentricity is considered, the trajectory of the end of each tooth $k$ is given by Equation 5, which is obtained when geometric radius $R_{k}$ is replaced by effective radius $R_{k e}$ in Equation 1.

$$
y_{i}=-\sqrt{\left[E \cdot \sin \theta_{k i}-\sqrt{R_{k i}^{2}-\left(E \cdot \cos \theta_{k i}\right)^{2}}\right]^{2}-x_{i}^{2}}
$$

Where $x_{i}$ is coordinate $x$ for a discrete point along axis $\mathrm{X}$, 
$y_{i}$ is the coordinate of each tooth $k$ end as a function of coordinate $x_{i}$,

$\theta_{k i}$ is the position angle corresponding to $x_{i}$ for a certain tooth $k$

In this way, the trajectory of each tooth $k$ end is determined. It is an arc around rotation centre $\mathrm{O}$ with variable radius $R_{e k}$. It corresponds to intersection between the milling tool tooth and workpiece material. Workpiece material is assumed to be in $y<0$.

\section{SIMULATION CONDITIONS}

Nominal diameter of the considered cylindrical tools was $6 \mathrm{~mm}$. All tools had 6 edges.

Three radius distributions were considered: $\mathrm{N}\left(2.995,0.001^{2}\right), \mathrm{N}\left(2.995,0.005^{2}\right)$ and $\mathrm{N}\left(2.995,0.010^{2}\right)$, corresponding to high quality, normal and low quality tools.

Likewise, three different eccentricity values were studied: $\mathrm{E}=0.001 \mathrm{~mm}, \mathrm{E}=0.005 \mathrm{~mm}$ and $\mathrm{E}=0.010 \mathrm{~mm}$ corresponding to low, medium and high eccentricity respectively. Five different feed per tooth values were taken into account: 0.02, 0.04, 0.06, 0.08 and $0.1 \mathrm{~mm}^{\text {tooth }^{-1}}$ revolution $^{-1}$.

In all cases, families of 100,000 tools were considered, since at higher number of tools the results do not significantly vary (Buj-Corral et al., 2013). None of the simulations accounted for the effects of any other possible causes of roughness, e.g. vibrations; tool flexion or tilt; or the plasticity of the material.

\section{MODEL VALIDATION}

\section{Experimental Setup}

The workpieces comprised steel blocks ( $70 \times 50 \times 40 \mathrm{~mm}$ ), each of which enabled four different experiments. In each experiment, a $40 \times 6 \mathrm{~mm}$ area of the block was machined 
by means of side milling. Three different materials were chosen: hardened steel (WNr 1.2344; hardness: HRC 52) for moulds; hardened steel (WNr 1.2379; hardness: HRC 62) for dies; and hardened stainless steel (WNr 1.2083; hardness: HRC 54) for molds.

Two cylindrical tools of the same manufacturer were employed. Tool diameter was 6 $\mathrm{mm}$ and number of edges was 6 . Tool overhang was $22 \mathrm{~mm}$. A Shrink fit holder DN40AD-CTH 20-75 with a DN40AD-SLK 12-45 collet (MST) was used. The machine was a Deckel Maho DMU 50 evolution high-speed (18,000 rpm) vertical machining centre with air blow for efficient chip removal. Cutting conditions were: $v_{c}=$ $215 \mathrm{~m} \cdot \mathrm{min}^{-1}$ for materials WNr. 1.2344 and $1.2083 ; v_{c}=180 \mathrm{~m} \cdot \mathrm{min}^{-1}$ for material $\mathrm{WNr}$. 1.2379; axial depth of cut $A d=6 \mathrm{~mm}$; radial depth of cut $R d=0.15 \mathrm{~mm}, f=0.01,0.02$, 0.03, $0.04,0.05$ and $0.06 \mathrm{~mm} \cdot$ tooth $^{-1} \cdot$ revolution $^{-1}$; cooling with air.

The errors of the tool edges due to the grinding processes and to the tool axis eccentricity were measured using a Marposs Quick Read digital indicator. The diameter of the tools was measured using a Parlec Series 1500 presetting machine. A Taylor Hobson Taylsurf Series 2 roughness stylus profilometer was employed for measuring roughness, using Taylor Hobson $\mu$ ltra software (v. 4.6.8).

\section{Experimental results for model validation}

Average radius of the two tools studied was $2.995 \mathrm{~mm}$. Average value of standard deviation $\sigma$ for radii for the two tools was was $2.1 \mu \mathrm{m}$ while average eccentricity value for the two tools was $6 \mu \mathrm{m}$.

Figure 3 shows experimental $\mathrm{Ra}$ and Rt values obtained for the three different materials (WNr 1.2344, WNr 1.2379 and WNr 1.2083). For each material and feed rate, 
roughness value corresponds to average value of roughness obtained with the two different tools. Experimental results were compared to corresponding simulated values for a distribution $\mathrm{N}\left(2.995,0.0021^{2}\right)$ with $\mathrm{E}=0.006 \mathrm{~mm}$. They were also compared to upper and lower reference values, i.e. maximum and minimum possible values for $\mathrm{Ra}$ and Rt according to tool and workpiece geometry (Equations 6 to 9).

Upper reference values correspond to the situation in which only one tooth will cut per revolution of the tool (Equations 6 and 7):

$$
\begin{aligned}
R_{a} \cong \frac{32 \cdot f_{n}^{2}}{R} \\
R_{t} \cong \frac{125 \cdot f_{n}^{2}}{R}
\end{aligned}
$$

Lower reference values correspond to the situation in which all edges are cutting (Equations 7 and 8):

$$
\begin{aligned}
& R_{a} \cong \frac{32 \cdot f^{2}}{R} \\
& R_{t} \cong \frac{125 \cdot f^{2}}{R}
\end{aligned}
$$
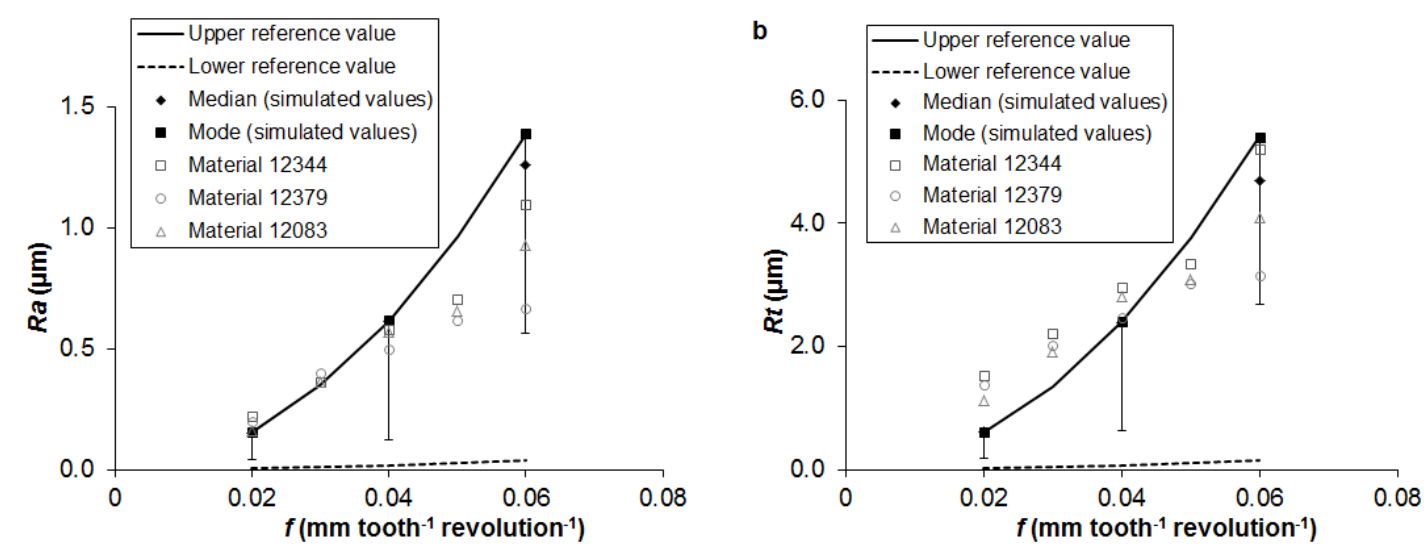

Figure 3 
At low feed per tooth values between $0.02 \mathrm{~mm}_{\text {tooth }}{ }^{-1}$ revolution ${ }^{-1}$ and $0.04 \mathrm{~mm}_{\text {tooth }}{ }^{-1}$ revolution $^{-1}$ both arithmetic average roughness (Ra) and peak-to-valley roughness (Rt) exceeded the upper reference value. This probably stems from the fact that simulations did not account for additional causes of roughness, for example vibrations or plastic deformation of the material.

For $f$ values higher than $0.04 \mathrm{~mm}^{\text {tooth }}{ }^{-1}$ revolution $^{-1}$, experimental roughness values fell to within the intervals obtained with the simulations.

Based on these comparisons, it was concluded that the model is valid for $f$ values higher than $0.04 \mathrm{~mm}_{\text {tooth }^{-1}}$ revolution ${ }^{-1}$.

\section{SIMULATION RESULTS}

First, histograms are presented for both $\mathrm{Ra}$ and $\mathrm{Rt}$, for low and high eccentricity values respectively. Later, confidence intervals for the median of Ra are depicted. Only results for $\mathrm{Ra}$ are presented since similar results were obtained for $\mathrm{Rt}$, for three different runout values, $\sigma=0.001 \mathrm{~mm}, \sigma=0.005 \mathrm{~mm}, \sigma=0.010 \mathrm{~mm}$, and two different eccentricity values, $\mathrm{E}=0.001 \mathrm{~mm}$ and $\mathrm{E}=0.010 \mathrm{~mm}$ respectively.

\section{Histograms}

Histograms for Ra and Rt, for $\sigma=0.005 \mathrm{~mm}$ and low eccentricity $\mathrm{E}=0.001 \mathrm{~mm}$, at $\mathrm{f}=$ 0.02 and $0.10 \mathrm{~mm}^{\text {tooth }}{ }^{-1}$ revolution ${ }^{-1}$ respectively are shown below, in Figure 4. 
$f=0.02 \mathrm{~mm} \cdot$ tooth $^{-1} \cdot$ revolution $^{-1}, \sigma=0.005 \mathrm{~mm}, E=0.001 \mathrm{~mm}$

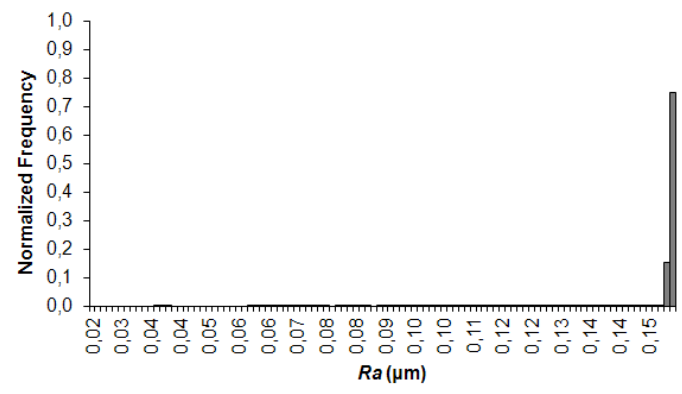

$f=0.1 \mathrm{~mm} \cdot$ tooth $^{-1} \cdot$ revolution $^{-1}, \sigma=0.005 \mathrm{~mm}, E=0.001 \mathrm{~mm}$

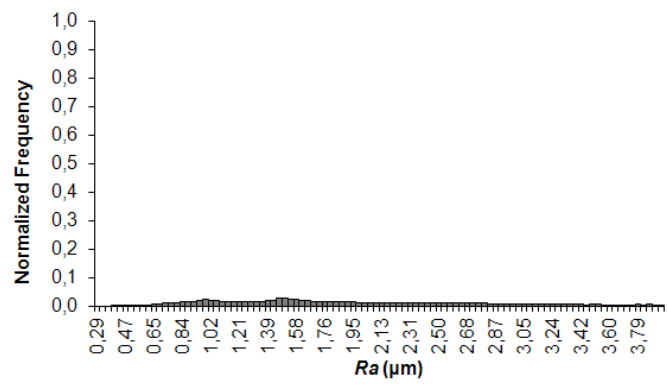

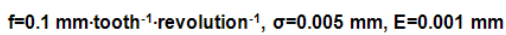

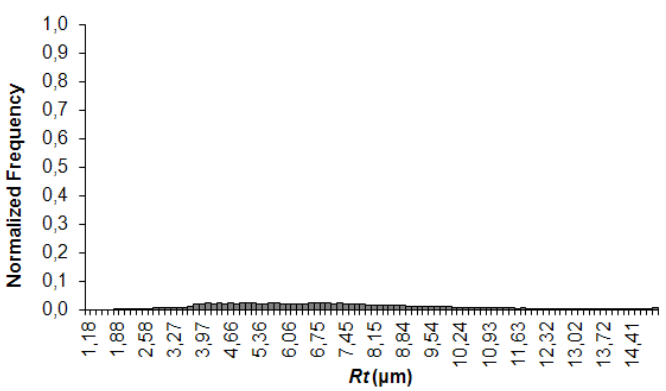

Figure 4

At lower feeds (Figures 4a and 4c), frequency distributions are highly asymmetric, and the modes of the roughness distribution are close to the upper reference value. In contrast, for higher feeds (Figures $4 \mathrm{~b}$ and $4 \mathrm{~d}$ ), the distributions are more symmetric, and the modes are not located close to the upper reference limit.

Histograms for Ra and Rt at $\sigma=0.005 \mathrm{~mm}$, high eccentricity $\mathrm{E}=0.010 \mathrm{~mm}$, and $\mathrm{f}=$ 0.02 and $0.10 \mathrm{~mm}^{\text {tooth }^{-1}}$ revolution $^{-1}$, are shown below, in Figure 5. 

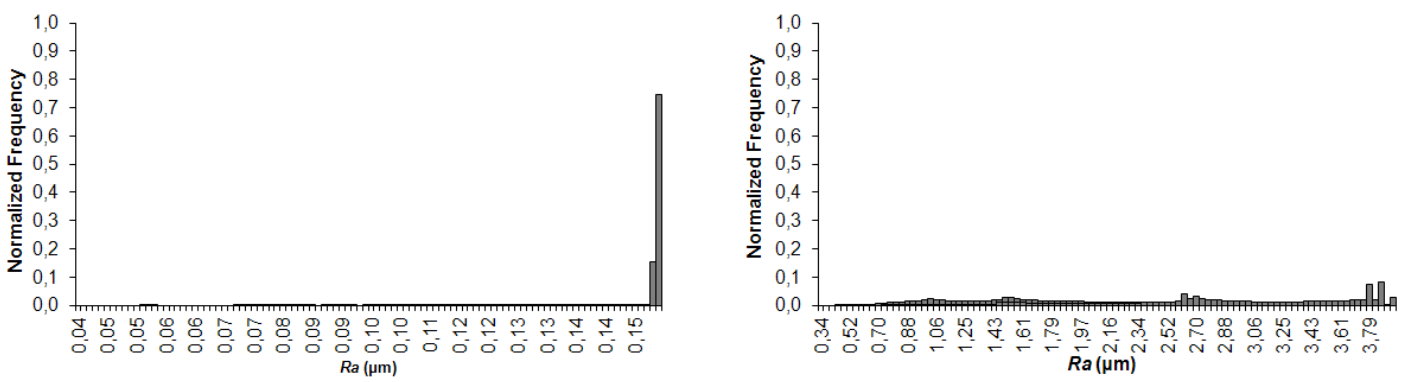

$f=0.02 \mathrm{~mm}^{\text {tooth }}{ }^{-1}$ revolution ${ }^{-1}, \sigma=0.005 \mathrm{~mm}, E=0.010 \mathrm{~mm}$

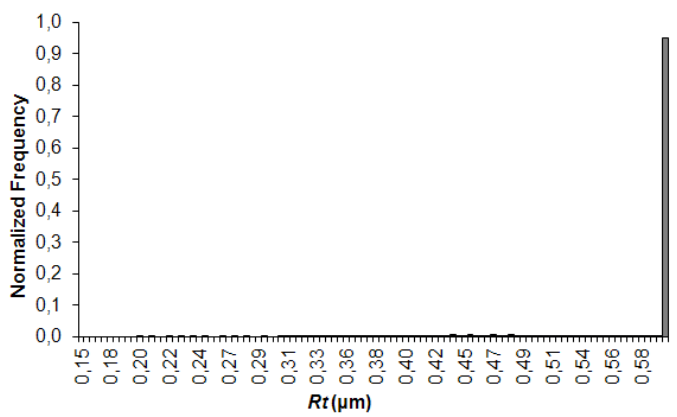

$\mathrm{f}=0.1 \mathrm{~mm} \cdot$ tooth $^{-1} \cdot$ revolution $^{-1}, \sigma=0.005 \mathrm{~mm}, E=0.010 \mathrm{~mm}$

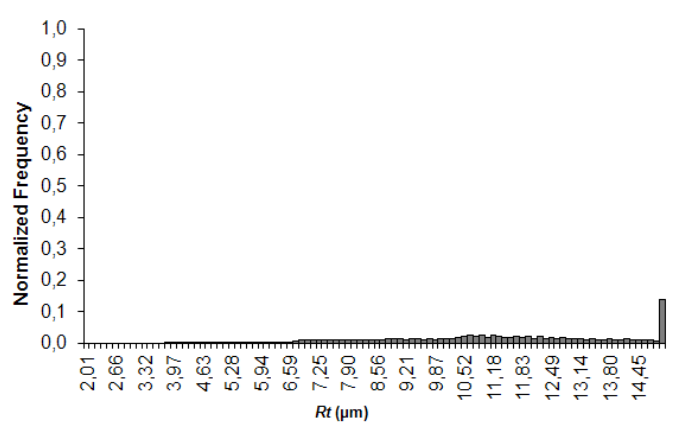

Figure 5

Histograms reveal that, at low feed, regardless of eccentricity, the mode roughness corresponds to the highest value possible (Figures 4a, 4c, 5a and 5c). At higher feeds, roughness depends on the eccentricity: low values of eccentricity give a broad range of roughness values of similar frequency, although the mode is far from the upper reference limit (Figures $4 \mathrm{~b}$ and $4 \mathrm{~d}$ ); and for high values of eccentricity the mode tends to be close to the upper reference value (Figures $5 \mathrm{~b}$ and $5 \mathrm{~d}$ ).

\section{Confidence intervals for the median of Ra}

Figures $6 \mathrm{a}$ and $6 \mathrm{~b}$ show the simulated values of Ra plotted against feed rate at $\sigma=0.001$ $\mathrm{mm}$ for $\mathrm{E}=0.001$ and $0.010 \mathrm{~mm}$ respectively. 

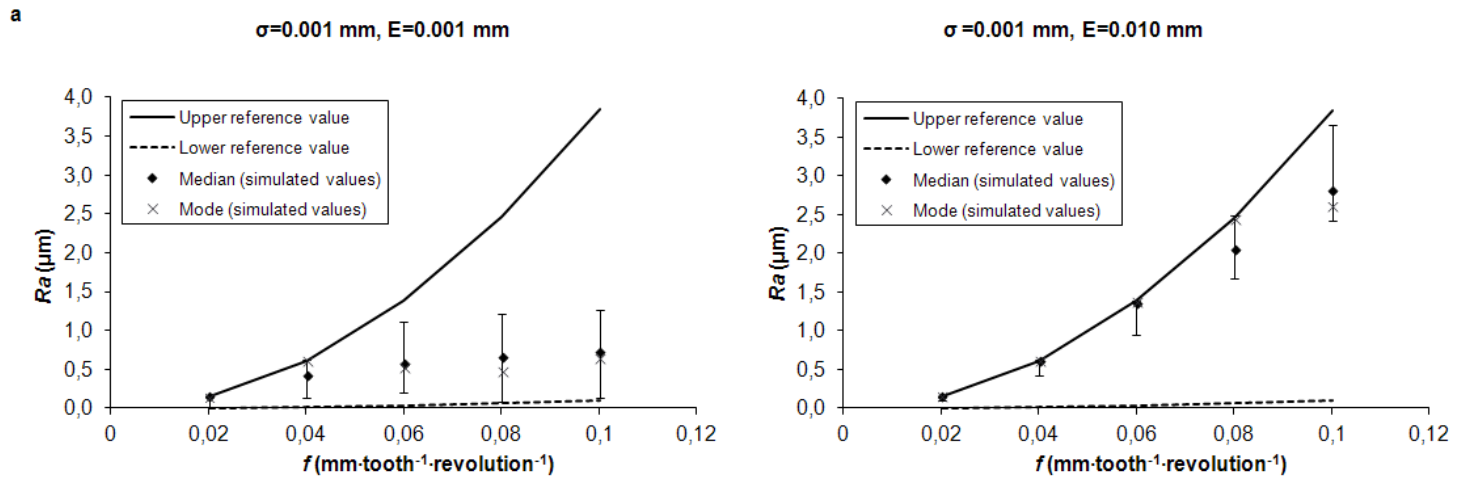

Figure 6

For $\sigma=0.001 \mathrm{~mm}$ quite narrow confidence intervals are obtained. At low eccentricity $E$ $=0.001 \mathrm{~mm}$ and low feed $f$, low roughness values are obtained. At high feed, the corresponding medians and modes are similar, regardless of feed considered, and confidence intervals remain narrow, since effective radii of teeth are similar and all of them will be likely to leave a mark on the workpiece's surface (Figure 6a). At high eccentricity $E=0.010 \mathrm{~mm}$, median and mode, as well as confidence intervals, get close to the high reference value (Figure $6 \mathrm{~b}$ ). This is due to the fact that, for high eccentricity, effective radius of one tooth tends to be higher than effective radii of the rest of the teeth leading to the situation where only one tooth will imprint the workpiece's surface.

Plots of simulated values of Ra against feed at $\sigma=0.005 \mathrm{~mm}$ are shown in Figures $7 \mathrm{a}$ and $7 b$. 

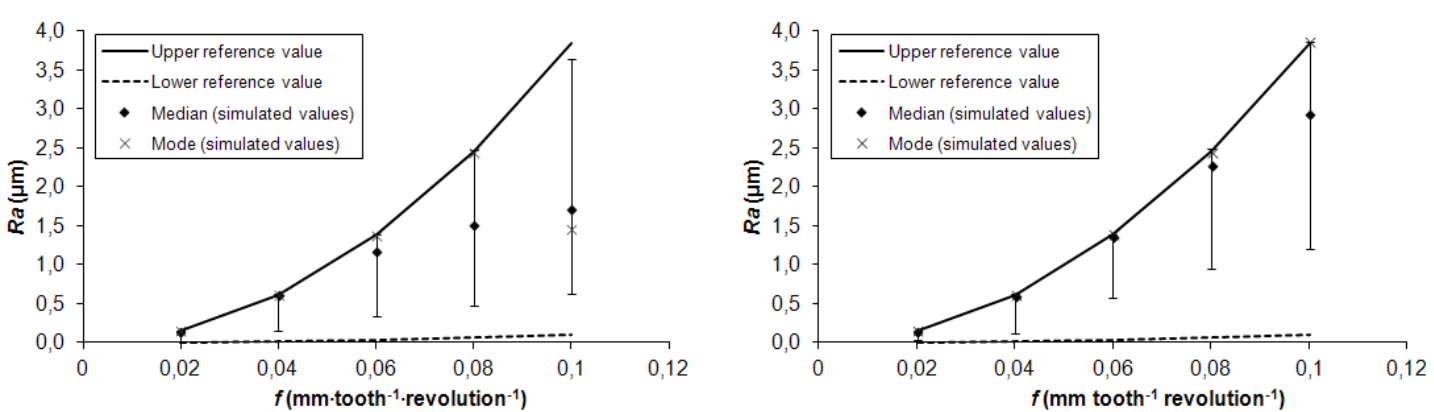

Figure 7

With this greater value of grinding error $(\sigma=0.005 \mathrm{~mm}$ instead of $0.001 \mathrm{~mm})$, the upper and lower limits of the confidence intervals for the median increase with increasing feed. For low eccentricity the median and the mode of the roughness distribution tend to get close to the upper reference value, especially at low feed values (Figure 7a). For high eccentricity, this tendency is enhanced. Since lower limit of confidence intervals increases with eccentricity, amplitude of confidence intervals decreases slightly with eccentricity (Figure 7b).

Plots of simulated values of Ra against feed for $\sigma=0.010 \mathrm{~mm}$ are shown in Figures 8a and $8 b$.

$\sigma=0.010 \mathrm{~mm}, E=0.001 \mathrm{~mm}$

$\sigma=0.010 \mathrm{~mm}, E=0.010 \mathrm{~mm}$
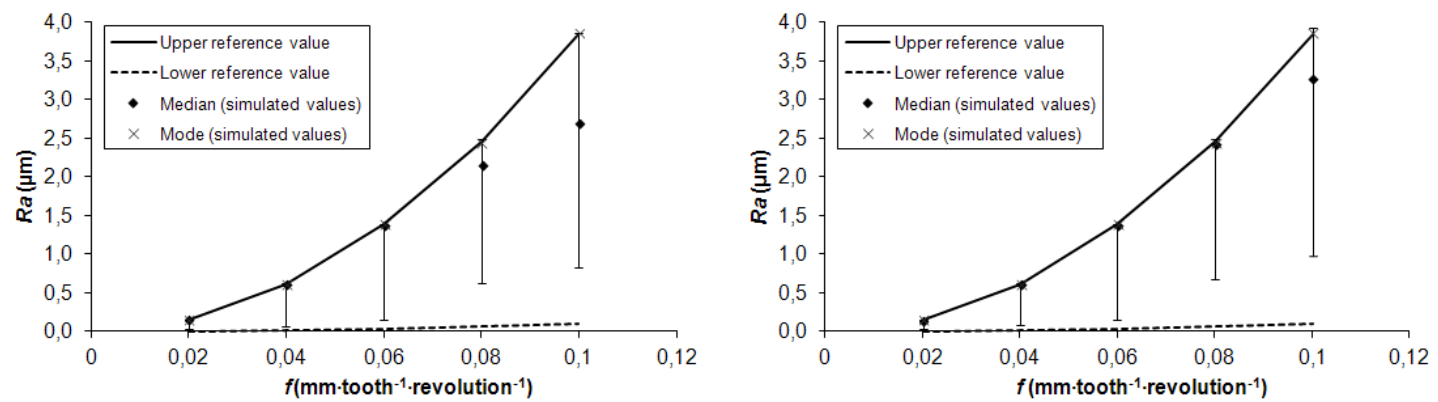

Figure 8 
At $\sigma=0.010 \mathrm{~mm}$, the highest studied value for grinding error, the mode coincides with the upper reference value for all eccentricity and feed values tested. At low eccentricity (Figure 8a) the median coincides with the upper reference value at low feed while at high feed it remains close to it. At high eccentricity (Figure $8 \mathrm{~b}$ ) the median almost coincides with the upper reference value at all feed considered.

In summary, width of confidence intervals increases with feed. This fact is more remarkable as runout due to grinding errors increases. Higher eccentricity slightly reduces width of confidence intervals. Both runout and eccentricity lead to higher median and mode values for the roughness distributions, which would ultimately coincide with the upper reference limit.

Figure 9 shows a contour plot for the mode of roughness distributions at different feed values and different eccentricity values, for low runout $\sigma=0.001 \mathrm{~mm}$ (Figure 9a) and high runout $\sigma=0.010 \mathrm{~mm}$ (Figure $9 \mathrm{~b}$ ).

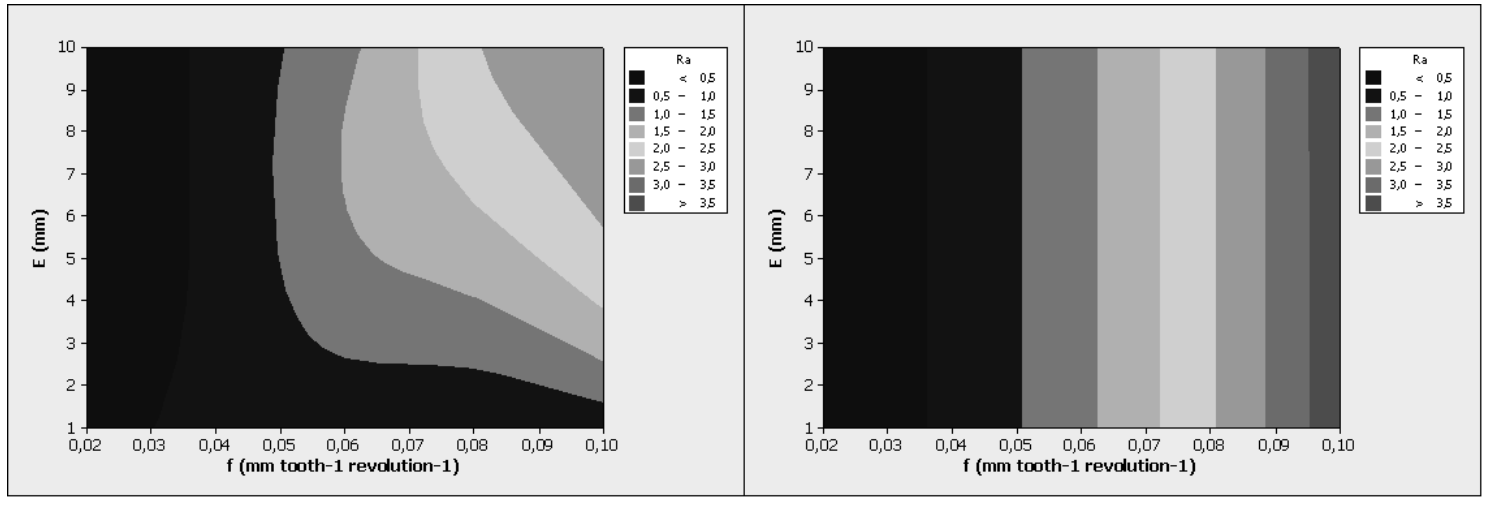

\section{Figure 9}

For low runout values, at low feed the mode of the roughness distribution remains almost constant with eccentricity, while at high feed, the mode of the roughness 
distribution increases significantly with eccentricity (Figure 9a). For high runout values, the mode of the roughness distribution does not depend on eccentricity but only on feed employed (Figure 9b).

It was concluded that, in side milling operations, eccentricity significantly influences roughness for tools having low runout. For tools having high runout, roughness mode is similar to upper reference value regardless of eccentricity value. For high runout only one tooth tends to leave a mark, and the mode of the roughness distribution is similar to upper reference value. For high eccentricity effective radius of one of the teeth will be higher than effective radius of the rest of the teeth leading to a similar behavior than for high runout. For this reason, for high runout effect of eccentricity is hidden.

\section{CONCLUSIONS}

Main conclusions of the paper are summarized as follows:

- For a family of tools having a certain runout value it was observed that, at low feed, shape of the roughness distribution is asymmetric and does not change significantly with eccentricity. On the contrary, at high feed, the higher eccentricity, the more asymmetric the roughness distribution becomes, and the closer the mode gets to the upper reference value corresponding to the situation where only one tool tooth will leave a mark per revolution of the tool.

- For a family of tools having low runout values, eccentricity strongly influences roughness, causing confidence intervals for the median, as well as the median and the mode to get closer to the upper reference value for roughness. This corresponds to the case of the workpiece being machined (or simply imprinted) by only one tooth. On the 
contrary, for a family of tools having high runout values, effect of eccentricity is not so important, since median and mode of the roughness distribution are yet closer to the upper reference value due to runout.

- Contour plots for the mode of the roughness distributions showed that, for high quality tools having low runout, for low eccentricity the mode of the roughness distribution will not remarkably increase with feed. On the contrary, for high eccentricity roughness will increase significantly with feed. For this reason, low eccentricity is recommended when high quality tools having low runout are employed, in order to obtain a good surface finish. On the contrary, for low quality tools having high runout, roughness mode depends basically on feed, regardless of tool eccentricity.

\section{ACKNOWLEDGEMENT}

The authors thank the Spanish Ministry of Education and Science for funding the project (DPI2007-66546) on which this article is based. They also thank Mr. Alejandro Domínguez-Fernández and Mr. Ramón Casado-López for their contributions to measurements and simulations.

\section{REFERENCES}

Altan, T., Lilly, B., Yen, Y.C., 2001. Manufacturing of Dies and Molds. CIRP Ann. Manuf. Technol. 50, 404-422.

Arizmendi, M., Fernández, J., Gil, A., Veiga, F., 2009. Effect of tool setting error on the topography of surfaces machined by peripheral milling. Int. J. Mach. Tools Manuf. 49, 36-52. 
Arrazola, P.J., Özel, T., Umbrello, D., Davies, M., Jawahir, I.S., 2013. Recent advances in modelling of metal machining processes. CIRP Ann. - Manuf. Technol. 62, 695718.

Buj-Corral, I., Vivancos-Calvet, J., González-Rojas, H., 2011. Influence of feed, eccentricity and helix angle on topography obtained in side milling processes. Int. J. Mach. Tools Manuf. 51, 889-897.

Buj-Corral, I., Vivancos-Calvet, J., González-Rojas, H., 2013. ROUGHNESS VARIATION CAUSED BY GRINDING ERRORS OF CUTTING EDGES IN SIDE MILLING. Mach. Sci. Technol. 17, 575-592.

Franco, P., Estrems, M., Faura, F., 2004. Influence of radial and axial runouts on surface roughness in face milling with round insert cutting tools. Int. J. Mach. Tools Manuf. 44, 1555-1565.

Fu, Y.C., DeVor, R.E., Kapoor, S.G., 1984. A Mechanistic Model for the Prediction of the Force System in Face Milling Operations. J. Eng. Ind. 106 (1), 81-88.

Ismail, F., Elbestawi, R., Du, R., Urbasik, K., 1993. Generation of milled surfaces including tool dynamics and wear. J. Eng. Ind. 115, 245-252.

Kline, W.A., DeVor, R.E., 1983. The effect of runout on cutting geometry and forces in end milling. Int. J. Mach. Tool Des. Res. 23, 123-140.

Kline, W.A., DeVor, R.E., Shareef, I.A., 1982. The prediction of surface accuracy in end milling. J. Eng. Ind. 104(3), 272-278.

Omar, O.E.E.K., El-Wardany, T., Ng, E., Elbestawi, M.A., 2007. An improved cutting force and surface topography prediction model in end milling. Int. J. Mach. Tools Manuf. 47, 1263-1275.

Ranganath, S., Sutherland, J.W., 2002. An improved method for cutter runout modeling in the peripheral milling process. Mach. Sci. Technol. 6(1), 1-20.

Ryu, S.H., Choi, D.K., Chu, C.N., 2006. Roughness and texture generation on end milled surfaces. Int. J. Mach. Tools Manuf. 46, 404-412.

Schmitz, T.L., Couey, J., Marsh, E., Mauntler, N., Hughes, D., 2007. Runout effects in milling: Surface finish, surface location error, and stability. Tehran Int. Congr. Manuf. Eng. (TICME2005), Tehran Int. Congr. Manuf. Eng. 47, 841-851.

Spiewak, S., 1995. An Improved Model of the Chip Thickness in Milling. CIRP Ann. Manuf. Technol. 44, 39-42.

Wang, J.-J.J., Zheng, C.M., 2003. Identification of cutter offset in end milling without a prior knowledge of cutting coefficients. Int. J. Mach. Tools Manuf. 43, 687-697. 


\section{FIGURE CAPTIONS}

Figure 1. Schematic representation of the side milling process, taking into account tool rotation speed of the milling tool $N\left(\mathrm{~min}^{-1}\right)$, feed rate of the workpiece $f_{r}\left(\mathrm{~mm} \cdot\right.$ revolution $\left.{ }^{-1}\right)$, axial depth of cut $A d(\mathrm{~mm})$, and radial depth of cut $R d(\mathrm{~mm})$. Source: (Buj-Corral et al., 2013)

Figure 2. Respresentation of the milling tool having eccentricity $E . \mathrm{C}$ is the geometric centre of the milling tool, $\mathrm{O}$ is the rotation centre and $\mathrm{P}$ the instantaneous position of tooth $k^{\text {th }}$ end.

Figure 3. Average measured roughness of three materials tested $(\mathrm{WNr} 1.2344, \mathrm{WNr}$ 1.2379 and $\mathrm{WNr} 1.2083$ ): (a) Ra; (b) Rt. Simulated intervals of roughness variation, from 100,000 simulation runs, median and mode were added for comparison

Figure 4. Histograms obtained from 100,000 simulations for $\sigma=0.005 \mathrm{~mm}$ and $\mathrm{E}=$ $0.001 \mathrm{~mm}$ : (a) Ra, with $\mathrm{f}=0.02 \mathrm{~mm}^{\text {tooth }}{ }^{-1}$ revolution $^{-1}$; (b) Ra, with $\mathrm{f}=0.1 \mathrm{~mm}^{\text {tooth }}{ }^{-1}$ revolution $^{-1}$; (c) Rt, with $\mathrm{f}=0.02 \mathrm{~mm}_{\text {tooth }^{-1}}$ revolution $^{-1}$; and (d) Rt, with $\mathrm{f}=0.1 \mathrm{~mm}$ tooth $^{-1}$ revolution ${ }^{-1}$

Figure 5. Histograms obtained from 100,000 simulations for $\sigma=0.005 \mathrm{~mm}$ and $\mathrm{E}=$ $0.010 \mathrm{~mm}$ : (a) Ra, with $\mathrm{f}=0.02 \mathrm{~mm}^{\text {tooth }}{ }^{-1}$ revolution $^{-1}$; (b) Ra, with $\mathrm{f}=0.1 \mathrm{~mm}$ tooth $^{-1}$ revolution $^{-1}$; (c) Rt, with $\mathrm{f}=0.02 \mathrm{~mm}_{\text {tooth }^{-1}}$ revolution $^{-1}$; and (d) Rt, with $\mathrm{f}=0.1 \mathrm{~mm}$ tooth $^{-1}$ revolution ${ }^{-1}$

Figure 6. Simulated Ra values plotted against feed rate: median, mode, interval, and upper and lower reference values at $\sigma=0.001 \mathrm{~mm}$ : (a) $\mathrm{E}=0.001 \mathrm{~mm}$, (b) $\mathrm{E}=0.010 \mathrm{~mm}$ 
Figure 7. Simulated Ra values plotted against feed rate: median, mode, interval, and upper and lower reference values at $\sigma=0.005 \mathrm{~mm}$ : (a) $\mathrm{E}=0.001 \mathrm{~mm}$, (b) $\mathrm{E}=0.010 \mathrm{~mm}$

Figure 8. Simulated Ra values plotted against feed rate: median, mode, interval, and upper and lower reference values at $\sigma=0.010 \mathrm{~mm}$ : (a) $\mathrm{E}=0.001 \mathrm{~mm}$, (b) $\mathrm{E}=0.010 \mathrm{~mm}$ Figure 9. Contour plots for the mode of $\mathrm{Ra}$ for different $\mathrm{f}$ values and different $\mathrm{E}$ values: (a) $\sigma=0.001 \mathrm{~mm}$, (b) $\sigma=0.010 \mathrm{~mm}$ 